\title{
ESR Studies on the Molecular Mechanisms of Fracture of Polymers at Low Temperatures
}

\author{
Takai Kawashima, ${ }^{*}$ Shigetaka Shimada, ${ }^{* *}$ Hisatsugu Kashiwabara, ${ }^{* *}$ \\ and Junkichi SoHmA
}

College of Engineering, Hokkaido University, Sapporo, Japan.

(Received November 29, 1972)

\begin{abstract}
From the ESR spectra observed from the mechanically-fractured polymers, such as PE, PP, PTFE, and PMMA, the radical species were identified. It was found that the radicals produced by the fracture of the polymers are the radicals formed from the scission of the carbon chains. Other characteristics of the radicals produced by the mechanical fracture of the polymer were found to be a high reactivity with oxygen and some anomalous behavior in the decay process. The molecular mechanisms of the fracture of the polymers were discussed on the basis of the facts established by ESR observation.
\end{abstract}

KEY WORDS ESR / Polymer Radicals / Mechanical Fracture / Molecular Mechanism /

It has been demonstrated by several researchers that ESR can give interesting information on the molecular mechanisms of fractures of bulk polymers. ${ }^{1-5}$ Identification of the radicals is important in such ESR studies and some trials have been reported ${ }^{1,2,6}$ on the identification of the radicals produced by mechanical fracture of polyethylene and polypropylene. However, the radical species were not firmly identified even for polyethylene and polypropylene and no trials were made for other polymers. And less attention was paid to the change of the radical specices with elevated temperatures, although such conversion of the radical species was commonly observed for polymer radicals produced by ionizing radiation. $^{7-9}$ In order to investigate on molecular mechanisms of the fracture of polymers it seems useful to identify the species of the radicals produced by mechanical fracture and also to follow the changes of the radicals with elevated temperatures.

\section{EXPERIMENTAL}

\section{Samples}

Polyethylene $(P E)$. The high density polyethylene, Sholex 6000, was used for the sample.

\footnotetext{
* Present address: Nissan Chemical Co.

** Nagoya Institute of Technology Nagoya Japan.
}

The powder of the purified sholex had been evacuated at $10^{-3} \mathrm{mmHg}$ for $20 \mathrm{hr}$ and was melted at $120^{\circ} \mathrm{C}$ for $10 \mathrm{hr}$. The PE rods (diameter about $5 \mathrm{~cm}$ ) were made of molten PE moulded in a glass tube.

Polypropylene $(P P)$. The isotactic polypropylene manufactured by Mitsui Chemicals was used as the sample. The PP rods were made by the method described for PE.

Poly(tetrafluoroethylene) (PTFE). Rods of poly(tetrafluoroethylene) made by Nihon Vulker were used as the samples.

Poly(methyl methacrylate) (PMMA). Rods of PMMA made by Mitsubishi Rayon Co. were used as the samples without any purification.

Polybutadiene $(P B)$. Two types of polybutadiene made by Japan Synthetic Rubber Co. were used; the trans-rich PB named Alfine (trans-1,4 70\%; cis-1,4 10\%; vinyl, 20\%) and the other named Diene (trans-1,4, 54.5\%; cis-1,4, 34.3\%; vinyl, 11.2\%). Blocks of purified Diene were used as the sample. Blocks of Alfine were used as the sample without purification.

ESR spectra were obtained by a 3BX-ESR spectrometer (JEOLCO) with $100-\mathrm{kHz}$ modulation. Care was used to avoid power saturation.

\section{Method of Mechanical Fracture}

The solid polymers immersed in liquid nitrogen in a vessel were cut with a saw and the 
sawdusts of the polymer was transfered under liquid nitrogen to an ESR sample tube. The sample tube containing the sawdust and liquid nitrogen was connected to the vacuum system and evacuated to $10^{-3}$ Torr, keeping the sample tube at $77^{\circ} \mathrm{K}$. By this procedure the saw dust of each polymer was produced at $77^{\circ} \mathrm{K}$ and preserved at the same temperature until the warming experiments. It should also be mentioned that the sawdust produced was not in contact with either air or oxygen, except for oxygen dissolved in the liquid nitrogen, during the sawing.

\section{SPECTRA AND IDENTIEICATION OF THE RADICAL SPECIES}

\section{Polyethylene}

The ESR spectra obtained from the mechani-

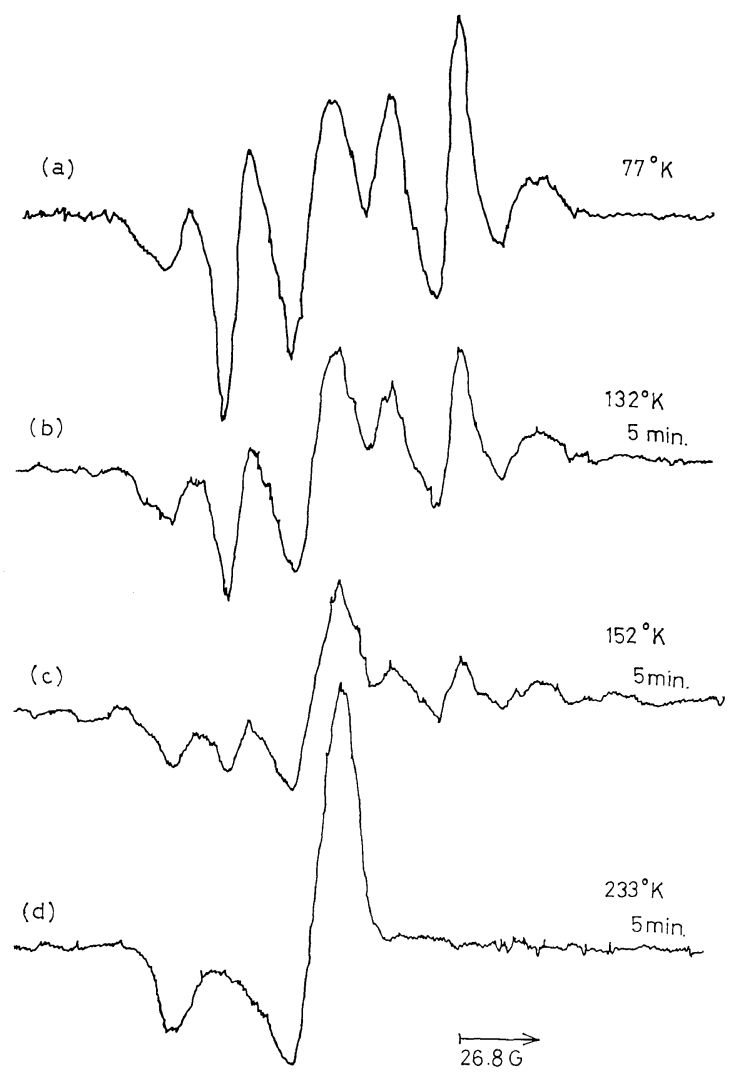

Figure 1. ESR spectra observed at $77^{\circ} \mathrm{K}$ from the mechanically-fractured polyethylene: (a) without any heat treatment; (b), (c), (d) after the heat treatment at the temperatures of $132^{\circ} \mathrm{K}, 152^{\circ} \mathrm{K}$, and $233^{\circ} \mathrm{K}$, respectively. cally fractured polyethylene are shown in Figure 1. All spectra were observed at $77^{\circ} \mathrm{K}$. The pattern " $a$ " is the spectrum without any heat treatment, and the spectra " $b$ ", "c"', and "dd" are those observed after heat treatment for $5 \mathrm{~min}$ at the temperatures $132^{\circ} \mathrm{K}, 152^{\circ} \mathrm{K}$, and $233^{\circ} \mathrm{K}$, respectively. Apparently the spectrum changes with elevated temperatures and this fact indicates that the majority of the radicals were changed in species by the heat treatment. The spectrum " $\mathrm{d}$ ", which is the spectrum of the final species of radical, is the well-known spectrum of the peroxyradical of polyethylene. ${ }^{10}$ The spectra " $a$ ", " $b$ ", and "cc" are all different in shape from the sextet spectrum ${ }^{7}$ of the $\mathrm{PE}$ radical produced by $\gamma$-irradiation. This means that the radicals produced by the mechanical fracture are different from the radiation-induced radicals. The spectrum " $a$ " in Figure 1 shows less symmetry at the central part, which suggests slight superposition of the spectrum of the peroxyradical, shown as "d" in Figure 1. Assuming this superposition, the spectrum of the peroxyradical was graphically substracted from the observed spectrum "a" and the symmetric spectrum shown as "a" in Figure 2 was obtained. This sextet spectrum is believed

(a)

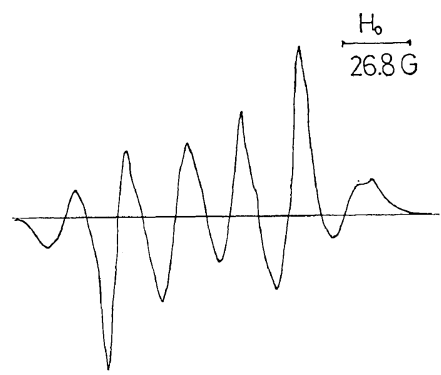

(b)

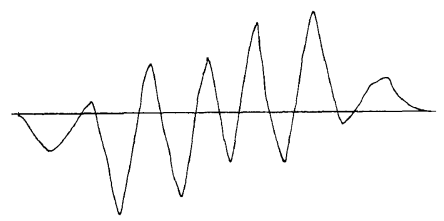

Figure 2. (a) ESR spectrum of the radical primarily produced by the fracture of polyethylene. (b) the simulated pattern on the assumption of the radical<smiles>CCC</smiles> 
to be the radical primarily produced by the mechanical fracture. The spectrum shown as " $b$ " in Figure 2 is the simulated one obtained

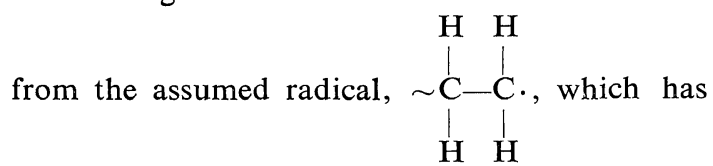
a coupling constant of 23 gauss with two $\alpha$-protons and one $\beta$-proton and of 46 gauss with another $\beta$-proton, and a line-width of 17 gauss. The fairly close agreement between these two spectra means that the radical responsible for the spectrum is $\sim \mathrm{C}-\mathrm{C} \cdot$. This identification is confirmed by the fact that the observed spectrum, "a" in Figure 2, is nearly identical to that from the radical $\mathrm{CH}_{3} \mathrm{CH}_{2} \mathrm{CH}_{2} \cdot$, which was produced by photolysis of the compound $\mathrm{CH}_{3} \mathrm{CH}_{2} \mathrm{CH}_{2} \mathrm{Br}^{11}$ This identification of the radical from the ESR spectrum convinced us that the primary radical produced by the mechanical fracture is the scission-type radical, $\sim \underset{\mathrm{H}}{\sim} \stackrel{\mathrm{C}}{\mathrm{C}}-\mathrm{C} \cdot \mathrm{H}$, which is formed by the scission of the carbon chain. The spectrum " $b$ " is a mixture of the two spectra, "a" and "c", and therefore the state which gives the spectrum " $b$ " is the intermediate state between "a" and "c"'. The spectrum "c" is mainly the octet and is partially superposed with the spectrum of the peroxyradical. The octet spectrum was identified as from the radical<smiles>CCCCCC</smiles>

The above identifications of the radicals lead us to the conclusion that the macroscopic destruction of polyethylene produces the scission of the polymer chain and the open bond of the chain carbon attracts a hydrogen from an adjacent site of the same molecule under slight heat treatment and finally the radicals are stabilized as the peroxyradical, probably either<smiles>CCCCCCCCCCCCO[O+]=O</smiles>

clusion is similar to what Butyagin and Radtsig mentioned, without detailed description, in their pioneer work. ${ }^{6}$ It is very interesting to note that no ESR spectrum was obtained from the nitric-acid-treated polyethylene even when it had been mechanically fractured.

\section{Polypropylene}

The ESR spectra which were obtained from the mechanically fractured polypropylene at $77^{\circ} \mathrm{K}$ are shown in Figure 3. The spectrum (b), which

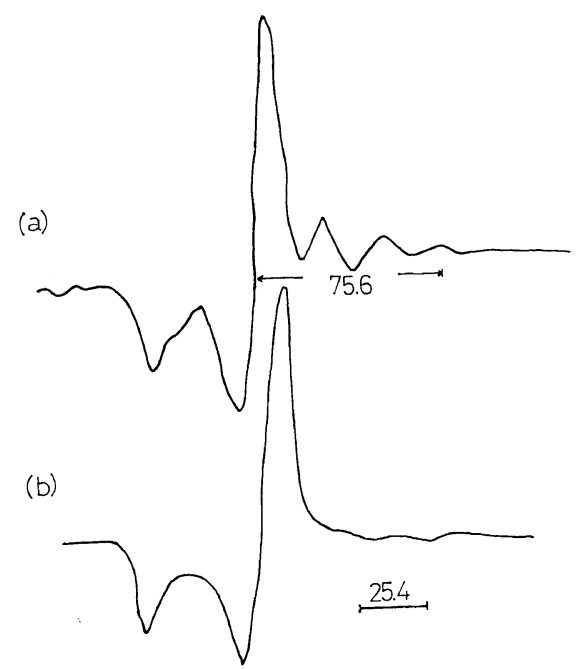

Figure 3. ESR spectra observed at $77^{\circ} \mathrm{K}$ from the mechanically-fractured polypropylene (a) without any heat treatment, (b) after the heat treatment at $156^{\circ} \mathrm{K}$.

is attributed to the peroxyradical, indicates that the primary radical was completely changed into the peroxyradical, even by the slight heat treatment at $156^{\circ} \mathrm{K}$ for $5 \mathrm{~min}$ in the case of PP. In the spectrum (a) obtained without any heat treatment one component of the spectrum is apparently from the peroxy radical. However, half of the spectrum of the primary radical appears without being greatly altered by the spectrum of the peroxy radical, as shown in Figure 3 a, and one may identify the radical from this surviving part of the spectrum. The half of the spectrum 
at the high field side appears as the four lines and the total spread of the spectrum is $152 \mathrm{G}$, which is nearly equal to seven times each separation of about $22 \mathrm{G}$. According to the analysis mentioned above the spectrum is taken as the octet with equal separation of $22 \mathrm{G}$, and this separation and the observed relative intensities of the four lines at the higher field side are same as the corresponding separation and intensities of the octet, which is the characteristic

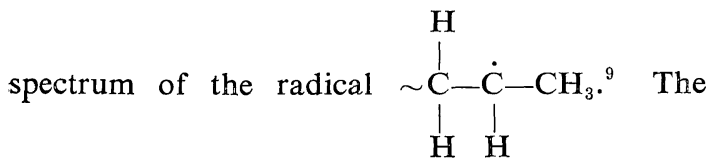
arguments presented above lead one to consider that most probable species of the primary radical produced by the mechanical fracture is the scission-type radical of polypropylene, $\sim \mathrm{C}-\mathrm{C}$. and that the primary radical of polypropylene is more reactive to oxygen than the PE radical. Poly(tetrafluoroethylene)

The pattern shown in Figure 4 is the spectrum observed at $77^{\circ} \mathrm{K}$ from the mechanically fractured

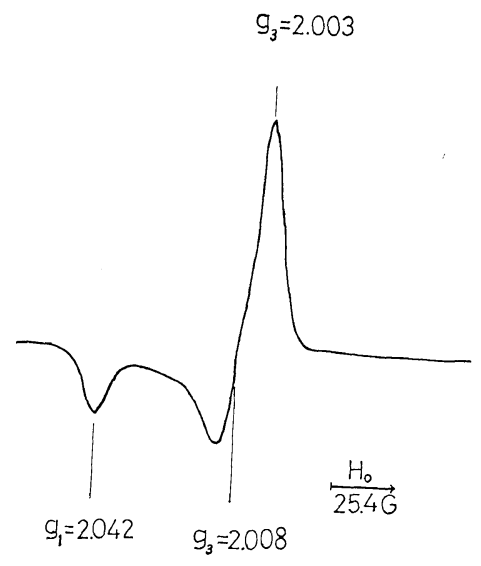

Figure 4. ESR spectrum observed at $77^{\circ} \mathrm{K}$ from the mechanically-fractured poly(tetrafluoroethylene).

PTFE without any heat treatment. The characteristic spectrum obtained indicates that the radical produced by the mechanical fracture under the conditions described in the section on Method of Mechanical Fracture had been completely converted into the peroxyradical, without heat treatment in the case of PTFE. Since the line shape of the peroxyradical of the

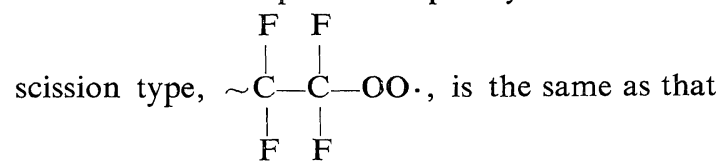
of the peroxyradical of polymer chain,

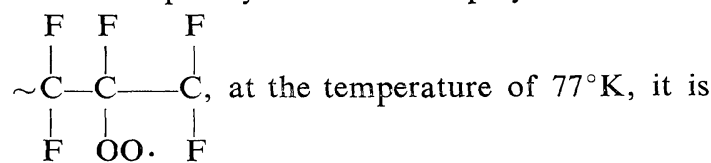
impossible to find out whether or not the radical responsible for this observed spectrum is the scission-type. However, the temperature variation of the spectrum of the peroxyradical is quite different depending on whether or not the attributed radical is the scission-type. ${ }^{12}$ The spectrum appears as a symmetric singlet at room temperature in the case of the scission radical, while the line-shape appears asymmetric for the chain radical. The details of the analysis is described in another paper. ${ }^{12}$ In the case of the peroxyradical produced by the mechanical fracture the spectrum at room temperature was found to be a singlet. On the basis of this it is believed that the radical produced by the mechanical destruction of PTFE is also the

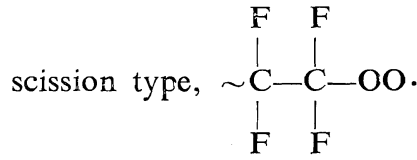

Poly(methyl methacrylate)

The spectrum observed from the mechanically fractured poly(methyl methacrylate) is shown in Figure 5. Although the spectrum is a little asymmetric, the half of the spectrum on the high field side is nearly the same in line shape and separations as the characteristic quintetquartet spectrum $^{13}$ of the radical species of

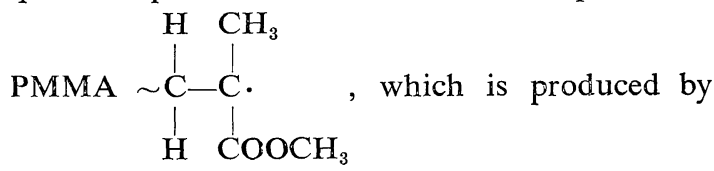
the scission of the $\mathrm{C}-\mathrm{C}$ chain. The lack of symmetry in the pattern is attributed to the slight superposition of the other spectrum which is presumably the spectrum of the peroxy radical, 
Molecular Mechanisms of Fracture of Polymers

Table I. The radical-species produced by the mechanical fracture

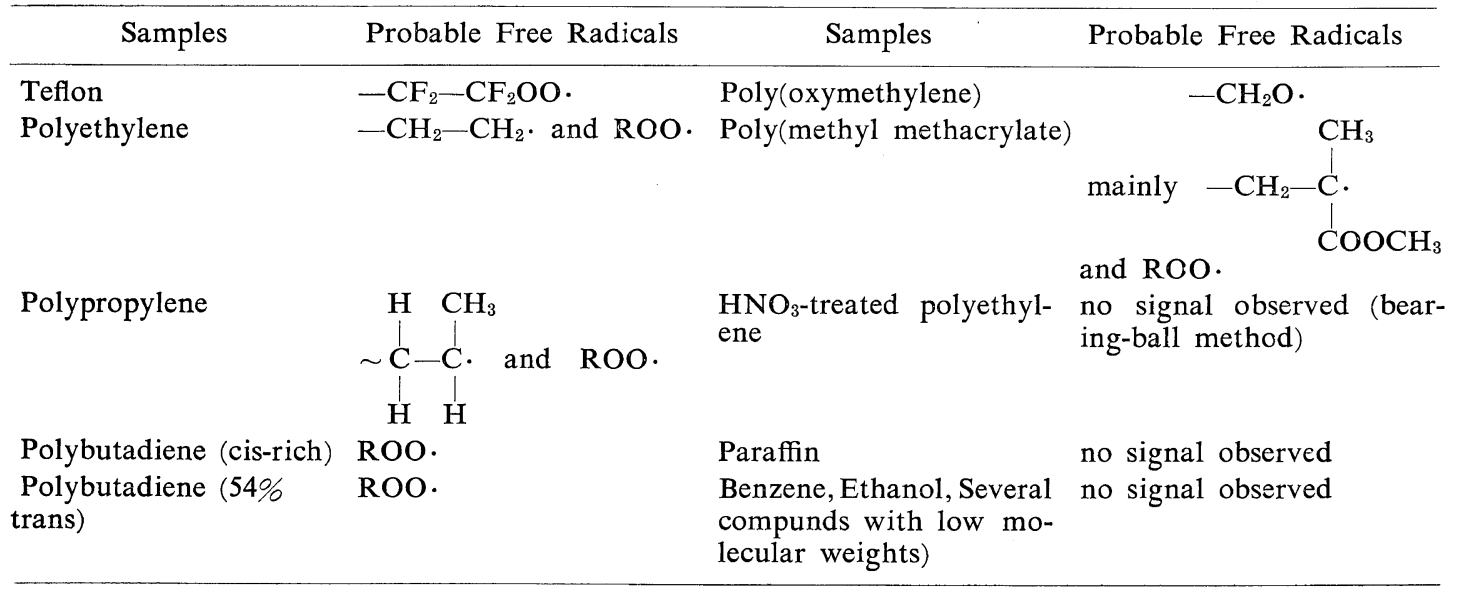

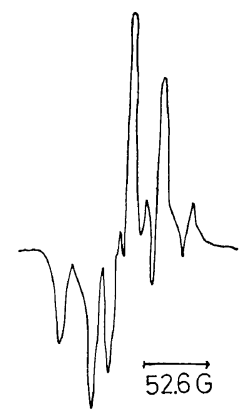

Figure 5. ESR spectrum observed at $77^{\circ} \mathrm{K}$ from the mechanically-fractured poly(methyl methacrylate).

because a superposition of the spectrum of the peroxy radical, as shown in the former spectra, disturbes mainly the low field side of the spectrum, as was the case for PP. From the above discussion one may say that the radical produced by mechanical fracture of PMMA is also the scission-type.

\section{Other Polymers}

The spectum observed from the radicals produced by the mechanical fracture of Diene was found to be the asymmetric spectrum of the peroxy radical, as for PTFE. From the other species of PB, Alfine, which was not purified, a quartet superposed with the spectrum of the peroxy radical was observed after the mechanical fracture. Although the radical responsible to the quartet is not definitely identified, the most plausible radical for this quartet is con- sidered to be the radical from the antioxidant in the Alfine matrix.

The spectrum observed from the mechanically fractured poly(oxymethylene) was that of the peroxy radical.

It is worth mentioning that the ESR spectra observed from these polymers after the mechanical fracture are different from those observed from the $\gamma$-irradiated polymers.

\section{Low Molecular Compounds}

By the same methods the solids of some low molecular compounds, such as benzene, ethanol, and paraffin were mechanically fractured at $77^{\circ} \mathrm{K}$; no ESR spectra were observed from these compounds. The radical species, which were produced by the mechanical fracture and identified by ESR spectra, are tabulated in Table I.

\section{DECAY CURVE}

The relative intensities of the ESR spectra observed at $77^{\circ} \mathrm{K}$ after heat treatment at various temperatures higher than $77^{\circ} \mathrm{K}$ for $5 \mathrm{~min}$ were plotted against the temperatures of the heat treatment. This curve is called the decay curve, since the ESR intensities decrease in most cases because of the decay of the radicals caused by the heat treatment at the elevated temperatures. The decay curve provides considerable information on the decay behaviors of the radicals, and thus were obtained for the radicals produced by the mechanical fractures of the polymers. 
Polypropylene

The decay curves are reproduced in Figure 6. The solid line is the decay curve obtained for

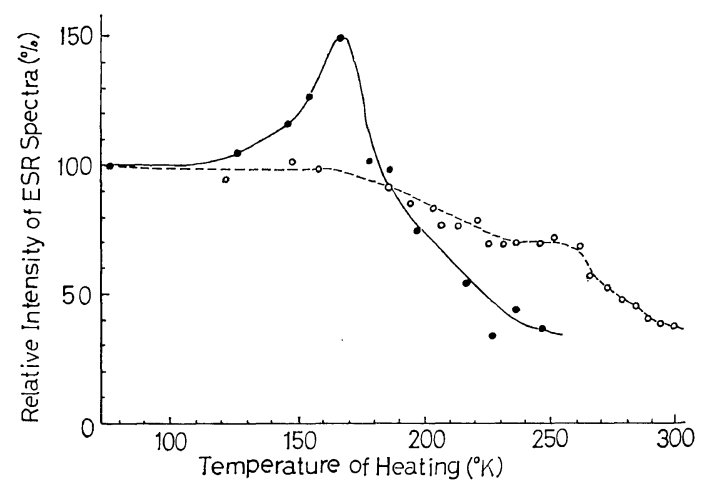

Figure 6. The decay curves of the PP radicals. The solid line for the radicals produced by mechanical fracture. The dotted line for the radicals produced by $\gamma$-irradiation.

the PP radicals, including the peroxy radicals, produced by the mechanical fracture and the dotted one is that for the PP radicals produced by $\gamma$-irradiation. One sees a clear difference between them in this comparison. The ESR spectra observed for the radicals produced by the mechanical fracture were found to be enhanced by the heat treatment at the temperatures between $100^{\circ} \mathrm{K}$ and $190^{\circ} \mathrm{K}$ instead of the usual behavior of a decrease of the spectral intensities for the radicals produced by irradiation. It was checked that the observed increase of the intensities was not a virtual one caused by the desaturation of ESR, but a real one caused by an increase of the total number of radicals. The decay curve for the mechanical fracture demonstrates that the radicals were freshly produced by the heat treatment alone over a range of temperatures.

\section{Poly(tetrafluoroethylene)}

The decay curves of PTFE are shown in Figure 7. In this case an increase of the radical number was also found for the mechanically fractured radicals, while no increase in the decay curve was found for the radicals produced by $\gamma$-irradiation.

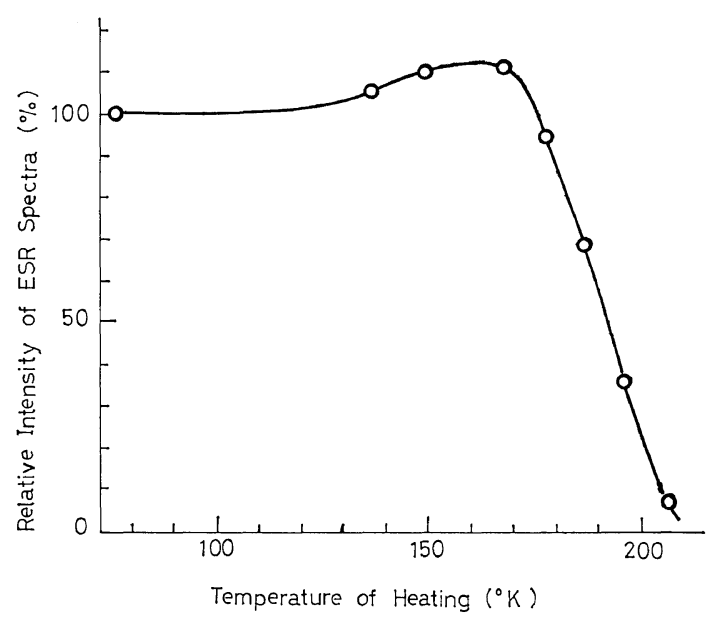

Figure 7. The decay curve of the PTFE radicals.

\section{Polyethylene}

The decay curve of polyethylene is known to be rather complex ${ }^{8}$ and shows three steps of the decay. One example of the decay curve of the radiation-induced radicals of $P E$ is shown as the dotted curve in Figure 8 and the solid-line curve in the same figure is the decay curve for the

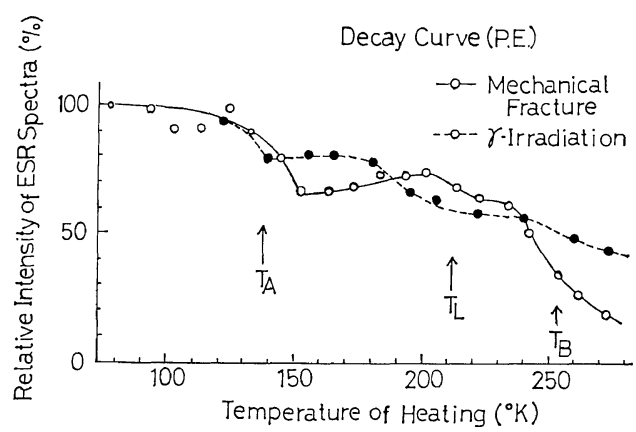

Figure 8. The decay curves of the PE radicals. The solid line for the radicals produced by mechanical fracture. The dotted line for the radicals produced by $\gamma$-irradiation.

radicals produced by the mechanical fracture. In the second step of the decay, which ranges from $150^{\circ} \mathrm{K}$ to $210^{\circ} \mathrm{K}$, an increase of the number of radicals was found in the case of the mechanical fracture.

Such an increase of the number of radicals was found for polybutadiene, although the relative amount of the increase was not so large 
as for PP and PTFE, but about the same as that of PE.

\section{DISCUSSION}

\section{Molecular Mechanism of Fracture of Polymers}

As described in the section on Spectra and Identification of The Radical Species, the radicals produced by the mechanical fracture of various polymers, PE, PP, PTFE, and PMMA, are all radicals of the scission-type. This fact means that the macroscopic fracture of polymers results in a microscopic rupture of the polymer chain, that is in the scission of $\mathrm{C}-\mathrm{C}$ bonds, at least for the above mentioned polymers. This formation of radicals of the scission-type makes an interesting contrast to the formation of chain radicals from the irradiated polymers. Also, the fact that no radical was formed from the mechanically fractured solid of the low molecular compounds leads us to believe that the rupture of the chemical bonds forming polymer chains caused by the macroscopic fracture of a polymer solid is a characteristic feature of polymers. Thus, one can expect to obtain some insight into the molecular mechanism of mechanical fracture through ESR studies of the radicals.

Molecular Mechanism of Fracture of Crystalline Polymers. Peterlin and his collaborators ${ }^{3,14,15}$ reported that ESR spectra were observed when crystalline polymers like Nylon and PE were greatly stretched and that the number of radicals obtained was dependent on the strain, but not on the stress. From these experimental results Peterlin ${ }^{3}$ proposed the model that the applied stress is mostly concentrated in the tie molecules which stretch to their maximum of possible length at the given strain, while increased tensile forces lead to a chain rupture, which produces free radicals. This molecular model of fracture contains an assumption that the crystalline blocks are sufficiently strong to remain unaffected in such a large deformation and that no polymer chain is pulled out from the crystalline part by the applied stress. It is reasonably assumed that a large deformation always preceeds the mechanical fracture. Assuming such a large deformation it is plausible that the bond rupture may happen on the tie molecules according to the Peterlin mechanism even in the mechanical fracture of polymers. The fact that no ESR spectrum was observed from the nitric-acid treated and fractured polyethylene supports our interpretation that the free radicals may be formed by the scissions of the tie molecules in the case of crystalline polymers like PE, PP, and PTFE.

Molecular Mechanism of Fracture of Amorphous Polymers. For amorphous polymers formation of radicals by mechanical fracture is not attributed to the rupture of the tie molecules, because no such classification of a crystalline or a tie molecule exists and no particular part of the polymer which is easily ruptured can be assumed in an amorphous polymer. The experiments demonstrate that the polymer chains are apparently ruptured, even in the case of an amorphous polymer like PMMA. Thus, a mechanism other than the Peterlin model is necessary for an explanation of the bond rupture of polymer chains in the amorphous polymer. Macroscopic fracture of a solid results from a large scale shearing motion of the constituents, such as grains or crystallites or molecules. In the case of a solid of low molecular compounds like benzene, the shearing motion of each molecule is independent of each other and a crack may grow, accompanied by a rupture of the van der Waals bonds between a particular molecule on which the stress is concentrated and the adjacent ones. This may be a reason why a radical is not formed by the mechanical fracture of the solids of the low molecular compounds. In a polymer solid, especially at a low temperature like $77^{\circ} \mathrm{K}$, the situation is different from a solid of the low molecular compounds. Large shearing displacement of single polymer, which leads to a macroscopic fracture, requires a simultaneous and incorporated motion of each constituent monomer, which corresponds to a single molecule in the solid of low molecular compounds. Thus, such shearing movement of a polymer needs more energy than that required for a molecule of low molecular weight. Let us take the activation energy, $E_{\eta}$, of viscosity of a low molecular compound as the approximate measure of the energy required for a shearing motion of a single monomer. Suppose $n$ is the number of monomers moving simultaneously in the situation where the monomers of a polymer interact with the monomers of the adjacent 
polymers in a way similar to the interaction between the molecules of low molecular weight. The energy which is needed for the large shearing displacement of a polymer molecule is $n$ times of the activation energy $E_{\eta}$ of the viscosity of the corresponding molecule of low molecular weight in the solid state. Ethane is considered to be a molecule similar to a monomer of polyethylene. To the best of the authors' knowledge no experimental data on activation energy is available for the viscosity of ethane in solid state at $77^{\circ} \mathrm{K}$. However, it is an empirical rule ${ }^{16}$ that the activation energy of viscosity is about $1 / 2.45$ of the molar energy of evaporation. Using this rule and the experimental value of molar energy of evaporation of ethane, $2.51 \mathrm{kcal} / \mathrm{mol},{ }^{17}$ $E_{\eta}$ for a monomer of polyethylene is assumed to be $(1 / 2.45)(2.51 \mathrm{kcal} / \mathrm{mol})=1.01 \mathrm{kcal} / \mathrm{mol}$. This consideration permits us to estimate the energy required for a shearing movement of a single polymer molecule as $n \times E_{\eta}$. On the other hand the bond energy of $\mathrm{C}-\mathrm{C}$ bond is known to be $83.1 \mathrm{kcal} / \mathrm{mol}$. Comparing the energy required for the large shear of a polymer, $n \times 1.01 \mathrm{kcal} /$ mol, we see that the energy required for rupture of single $\mathrm{C}-\mathrm{C}$ bond is smaller than that needed for the large shear of a polymer if $n$ is larger than 83. The estimate, although it is rough, leads to a conclusion that less energy is consumed for the rupture of single $\mathrm{C}-\mathrm{C}$ bond than for the simultaneous shearing displacement of a sufficiently long polymer in a solid. In such a case the crack may proceed by breaking the $\mathrm{C}-\mathrm{C}$ bond instead of shearing the polymer molecule, and a radical of scission-type may be produced by macroscopic destruction. The argument presented above helps us to understand the reason why bond fracture was not observed for the paraffin, whose carbon number is less than 80. As described in the former section the Peterlin model involves the assumption that no polymer molecule is pulled out from a crystalline. This assumption has support from the discussion in this section, because the folding period of a crystallite is, generally larger than $100 \mathrm{~A}$, and the number of monomers moving simultaneously under the stress is sufficiently large to prevent the shearing motion of a polymer in the crystallite.

\section{Reactivity to Oxygen}

One of the characteristic features of the radicals produced by the mechanical fracture is their high reactivity to oxygen. The radicals produced from the same polymers by irradiation either stay unchanged or easily decay before they convert into the peroxy radical, at a temperature of $77^{\circ} \mathrm{K}$ or a little higher. This high reactivity may be explained by the easier accessibility of the oxygen molecule to the radicals which exist on the surface of the fragment produced by the mechanical fracture.

\section{Anomalous Behavior of Decay Curves}

The decay experiments of the radiation-induced radicals demonstrate that the radicals decay with increasing temperature for $\mathrm{PE},{ }^{8} \mathrm{PP},{ }^{7} \mathrm{PTFE},{ }^{18}$ and $\mathrm{PB}^{19}$. This is normal behavior for the radicals, because the radicals are unstable species and easily decay out. The decay curves of the radicals produced by the mechanical fracture show, more or less an additional production of radicals by the heat treatments for the polymers dealt with here. This is anomalous behavior which seems to be characteristic for the mechanical fractures of the polymers. At present we are not yet able to explore the reasons for this anomalous behavior.

\section{REFERENCES}

1. P. Yu. Butyagin, I. V. Kolbanev, and V. A. Radtsig, Soviet Phys. Solid State, 5, 1642 (1964).

2. T. Kawashima, M. Nakamura, S. Shimada, H. Kashiwabara, and J. Sohma, Rept. Prog. Polym. Phys. Japan, 12, 469 (1969).

3. O. D. Campbell and A. Peterlin, Polym. Letters, 6, 481 (1968); A. Peterlin, J. Polym. Sci. Part C, No. 32, 297 (1969).

4. H. H. Kausch, J. Macromol. Sci. Chem., C4, (2), 243 (1970).

5. K. L. De Vries, J. Polym. Sci. Part C, No. 32, 325 (1971).

6. V. A. Radtsig and P. Yu. Butyagin, Polym. Sci. (USSR), A9, 2883 (1967).

7. S. Nara, H. Kashiwabara, and J. Sohma, $J$. Polym. Sci., Part A-2, 929 (1967).

8. S. Nara, S. Shimada, H. Kashiwabara, and J. Sohma, ibid., Part A-2, 6, 1435 (1968).

9. S. Shimada, H. Kashiwabara, and J. Sohma, ibid. Part A-2, 3, 1291 (1970).

10. For example, K· Tsuji and T. Seiki, Polymer J. 2, 606 (1971). 


\section{Molecular Mechanisms of Fracture of Polymers}

11. P. B. Ayscough and C. Thomson, Trans. Faraday Soc., A-58, 1477 (1962).

12. S. Moriuchi, M. Nakamura, S. Shimada, H. Kashiwabara, and J. Sohma, Polymer, 11, 630 (1970).

13. H. Fischer, Polymer Lett., 2, 529 (1964).

14. A. Peterlin, J. Polym. Sci., Part A-2, 7, 1151 (1969).

15. G. S. P. Verma and A. Peterlin, J. Macromol. Sci. Phys., B4, (3), 589, (1970) and Kolloid S. \& S. F. Polymere, 236, 111 (1970).
16. J. O. Hirschfelder, C. F. Curtis, and R. B. Bird, Molecular Theory of Liquids, John Wiley and Sons, New York, N. Y. 1940 p. 623.

17. Landort-Bornstein Tabelle, 6 Auf. II Band, 4 Teil.

18. T. Kawashima: Master's Thesis, College of Engineering, Hokkaido University (1968).

19. N. Tsuchihashi, S. Shimada, H. Kashiwabara, and J. Sohma, Rept. Prog. Polym. Phys. Jap. 12, 461 (1969). 